\title{
Strong lensing studies with the 3.6-m Devasthal Optical Telescope: opportunities and challenges
}

\author{
Dominique Sluse*
}

STAR Institute, Quartier Agora - Allée du six Août, 19c B-4000 Liège, Belgium

\begin{abstract}
Strongly-lensed quasars and active galactic nuclei (AGN), namely active galaxies that appear multiply imaged due to a foreground lensing galaxy, are exceptional astrophysical tools that may be used to probe the expansion rate of the Universe, study the evolution of galaxies and of their dark matter content over cosmic time, and zoom in into the structure of AGNs. We are entering a new era in observational astrophysics, no longer limited by the number of accessible targets, but by our ability to carry out follow-up observations. We list several science cases where the DOT and other Belgo-Indian telescopes may provide crucial high-quality data: (1) Photometric monitoring of strong lenses for cosmography (2) Confirmation of new strongly-lensed candidates in the era of big surveys (3) Advanced study of the structure of AGN using the technique of gravitational microlensing. For each of these topics, we provide a brief scientific overview of the technique and scientific question, and quantify the observational requirements for successful programs.
\end{abstract}

Keywords: gravitational lensing - strong-lensing - observations - time-delay - cosmology

\section{Introduction}

The phenomenon of gravitational lensing (GL), namely the deflection of light from a distant source when passing in the vicinity of a foreground mass, is a major tool of modern astrophysics. Of particular interest is strong gravitational lensing produced by a galaxy-scale deflector where multiple images of a background active galactic nucleus (AGN) are observed. Those cosmic mirages are powerful astrophysical laboratories that may be used to address various questions on the origin and evolution of the Universe and its constituents. Since a few decades, GL ceased being a fancy curiosity to become a popular working technique providing the most accurate measurement of the mass of distant galaxies, a natural telescope to observe high-redshift galaxies, an innovative tool to constrain the structure of AGNs, and a powerful cosmological probe. About one over seven hundreds AGNs is multiply imaged by a foreground galaxy. Since the discovery of the first gravitationally lensed quasar 40 years ago (Walsh et al. 1979), more than 200 hundred new systems have been discovered (Figure 1), half of them in the last 5 years owing to emergence of new lens-finding techniques and development of large space and ground-based surveys (Agnello et al. 2015, Ducourant et al. 2018, Delchambre et al. 2019, Lemon et al. 2019). The future of gravitational lensing-based research is bright, with the launch in 2021 of the space mission Euclid, that is the first major space mission to be mainly driven by GL-related science, but also upcoming operation of numerous surveys, that will increase by orders

*dsluse@uliege.be 
of magnitude the number of known strongly-lensed objects (Oguri \& Marshall 2010, Agnello et al. 2015). In the following sections, we discuss opportunities for 2-m and 4-m class telescopes to play a key role in strong-lensing related science.

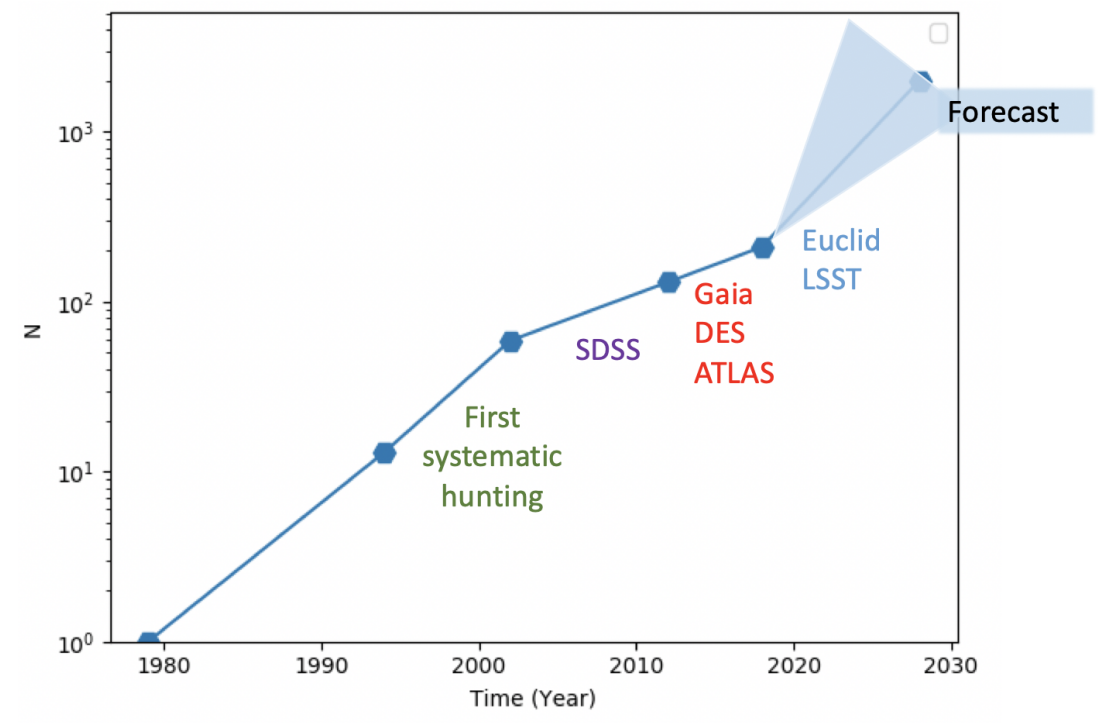

Figure 1: Number of confirmed gravitationally lensed quasars as a function of time.

\section{Expansion rate of the Universe}

Science Rationale: Achieving sub-percent accuracy on the measurement of $H_{0}$ is one of the main goal of cosmological studies in the coming decade. The value of $H_{0}$ has long been controversial but now, several techniques, alone or in combination, measure $H_{0}$ to better than $5 \%$ precision: SNIa and Cepheid stars (along with secondary calibrations), Baryon Acoustic Oscillations (BAO), and the Cosmic Microwave Background. Note that the latter does not measure $H_{0}$ on its own unless perfect flatness is assumed, and requires to be combined with other probes to effectively constrain $H_{0}$ with high precision. Two other probes promise high precision in the future: water megamasers and gravitational waves. While water megamasers is expected to reach a few percents accuracy in the coming 5 years (Gao et al. 2016), gravitational waves are not expected to provide tight constraints on $H_{0}$ before a decade (Abbott et al. 2017), as a large number of events is required to derive percent accuracy on $H_{0}$.

The gravitational time delay technique (hereafter TD; Refsdal 1964), consists in obtaining light curves of the individual images of a lensed AGN. Since those images are from the same source, their light curve is basically the same but a shift in time occurs due to the difference in light paths and gravitational potential of the lens. Long-term deformations of the light curves sometimes occur in one of the images due to gravitational lensing produced by the stars in the galaxy, an effect called microlensing. Because the time delay is mostly proportional to $H_{0}$, the precision on the time delay measurement governs the final accuracy on the derived value of $H_{0}$. Time delays can nowadays be measured down to $3 \%$ relative accuracy, the main uncertainties arising from the time sampling and accuracy of the light curve and from microlensing (Bonvin et al. 2016, Tie \& Kochanek 2018). Nowadays, TD allows one to measure $H_{0}$ to better than $4 \%$ accuracy (Bonvin et al. 2017, Birrer et al. 2019). Because of its characteristics, the TD technique is a nice complement of the abovementioned methods, and plays a key role in modern cosmology.

Observational constraints: A critical ingredient of the TD method is the ability to measure timedelays with an accuracy $<3 \%$ for each lens system. The COSMOGRAIL project (Eigenbrod et al. 
2005) has demonstrated that this is achievable with 1-m class telescopes performing weekly monitoring over typically a decade (e.g. Bonvin et al. 2017). It is now demonstrated that the same precision can be achieved with a few seasons of daily monitoring capturing rapid variability of a few millimagnitudes (mmag; Courbin et al. 2018). Such a high precision on photometry requires larger telescopes, such as $2 \mathrm{~m}$-class or $4 \mathrm{~m}$-class telescopes, but also good seeing conditions (i.e. typically $\leq 1.2^{\prime \prime}$ ) as lensed images are rarely separated by more than $2^{\prime \prime}$. A spatial sampling of the image of at least $0.35^{\prime \prime} /$ pixel (but $0.2^{\prime \prime} /$ pixel is optimal) is also needed to ensure Nyquist PSF sampling under good seeing conditions and enable image and lensing galaxy deblending. Telescopes of the COSMOGRAIL network fulfil those technical requirements. Based on those data, we demonstrated that accurate deblending of the lensed images is achieved using advanced image deconvolution techniques, such as the MCS technique (Magain, Courbin, Sohy 1998). These conditions being fulfilled, it is possible to reach mmag accuracy on the photometry of a 18.5 mag lensed images with an exposure time of $4 \times 320 \mathrm{~s}$ on a $2-\mathrm{m}$ class telescope, as needed for high precision time delays. By doubling the exposure time, it has been shown that $\sim 3 \mathrm{mmag}$ rms is achievable on the photometry of a lensed quasar with $R_{c} \approx 20.5 \mathrm{mag}$ on a 2-m class telescope (Courbin et al. 2018). With the DOT, and the same observational setup, we may expect to reach the same photometric accuracy for an object typically $\sim 1.6$ mag deeper than quoted above.

\section{Confirming lensed AGN candidates}

Science Rationale: An increase by more than an order of magnitude of the number of lensed AGNs is expected in the next decade (e.g. Oguri et al. 2010), but discoveries will be based mostly on multi-colour imaging. Even if the level of false positive falls below $10 \%$ in the next decade, there would still be several thousands of systems to be confirmed with spectroscopy, and a few hundreds with BINA telescopes (i.e. down to $i \sim 20.5 \mathrm{mag}$ ). Obtaining spectroscopy of the different lens components is not only important to confirm GL candidates, but is also instrumental to most of the astrophysical applications of gravitational lensing. In particular, an accurate redshift of the lens and source are required for cosmographic inference (Section 2). Spectroscopic data, with a minimum resolving power $\mathcal{R} \equiv \lambda / \Delta \lambda \geq 300$, also offer a simple diagnostic of the presence of microlensing in the lensed images (e.g. Sluse et al. 2012, Sluse et al. 2015).

Observational constraints: As for photometric monitoring projects, the spectroscopic confirmation of lensed quasar candidates requires good seeing conditions and spatial sampling (better than $0.35 \%$ / pixel to enable deblending of spatially overlapping spectra). In order to maximize the success in measuring the redshift of the lens and source, a large spectroscopic coverage is required (i.e. the whole visible range and/or near- Infrared range), while a resolving power as low as $\mathcal{R}=200$ is sufficient for redshift measurements. An integral field spectrograph (IFS) would be well-suited for such an application, but the characteristics of DOTIFS (Chung et al. 2018), the currently planned IFS for the DOT, may not be optimal for that purpose due to its large spatial sampling. Instead, standard long-slit spectrograph should fulfill most needs. A setup allowing for multi-object spectroscopy, with slits placed on the lensed system and on field stars, would be ideal as it allows one to apply spectral deconvolution techniques (Courbin et al. 2000) and to accurately separate the lensed images from the lensing galaxy (e.g. Eigenbrod et al. 2006).

\section{Scanning AGNs with microlensing}

Science Rationale: Cosmic microlensing uses the deflection of the light produced by stars in a strong- 


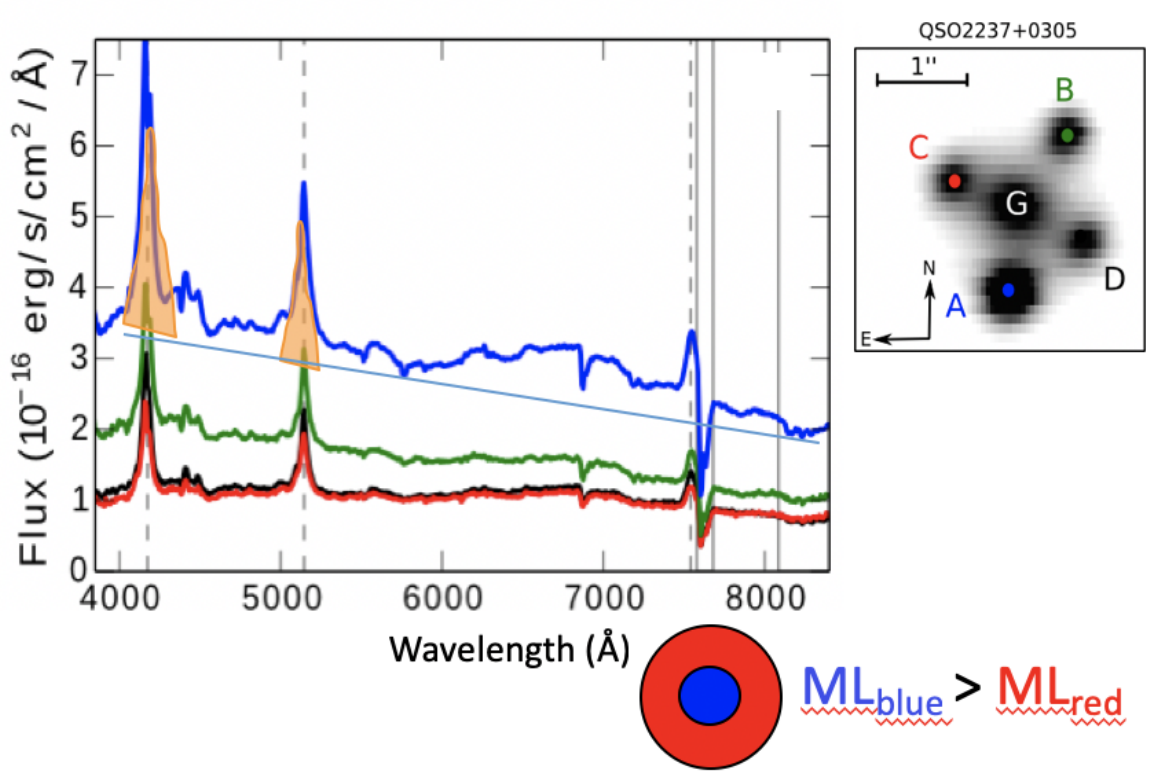

Figure 2: Spectra (observed reference frame) of the lensed images A-B-C-D of the gravitationally lensed AGN Q2237+0305 (from Braibant et al. 2016). This illustrates the impact of microlensing on AGNs' spectra. Image A is magnified due to microlensing compared to the other lensed images. We clearly notice a chromatic change of the continuum emission in that image, with the bluest wavelengths being more magnified than the reddest ones, yielding a steeper apparent slope of the continuum emission of image $A$. This happens because of the more compact (and thus more microlensed) region emitting the bluest continuum wavelengths (sketch on the bottom right). Detailed analysis shows that the broad $C$ IV and CIII ] emission lines (colored in orange) are also magnified due to microlensing. Such data allow one to constrain the size and the geometry of those regions.

lensing galaxy to scan AGNs otherwise unresolved with classical instrumentation. The chromatic microlensing of the UV-continuum emission of AGNs is commonly detected with ground-based data, either using multi-band imaging or spectroscopy. It has yielded the best measure of the temperature profile of the accretion disc (i.e. $20 \%$ precision on the slope in Eigenbrod et al. 2008a). Similar constraints on the disc temperature could only be achieved recently with a more direct technique that measures the time lags between multi-band flux variations of a few local AGNs (e.g. Fausnaugh et al. 2018, Edelson et al. 2019). Those results confirm the oversize of the accretion disc unveiled by microlensing studies (e.g. Morgan et al. 2010, Blackburne et al. 2010), and suggest that the standard Shakura-Sunyaev accretion disc model needs to be revised. The region giving rise to broad emission lines is also susceptible to cosmic microlensing. Microlensing-induced line deformation are ubiquitous among strongly-lensed AGNs (Sluse et al. 2012), and can be used to constrain the size and (yet poorly characterized) geometry of this region for different emitting gas species (Braibant et al. 2016, Braibant et al. 2017).

Observational constraints: Cosmic microlensing may be based on single- or multi-epoch data. Only low precision constraints on the properties of an AGN (e.g. temperature profile of the disc, size of the broad line region) may be retrieved from the analysis of its single-epoch data. Therefore, this approach requires to study a large sample of objects from which average information about the population is inferred. While the study of the accretion disc can be performed with broad-band imaging (ideally from 4000 to $25000 \AA$ ), , more accurate chromatic changes are derived based on spectroscopy. 
Table 1: Summary of important science opportunities for the DOT and other BINA telescopes. Columns 1 and 2 provide the nature of the project and of the data sets, column 3 gives an estimate of the exposure time. The last column lists additional observational requirements; with $R$ referring to $R$-band magnitude, and $\mathcal{R}$ referring to the resolving power of the spectrograph.

\begin{tabular}{|c|c|c|c|}
\hline Project & Data & Exp. time & Comment \\
\hline Time-delay & Imaging & $4 \times 640 s$ & $\begin{array}{l}2 \text {-m class, daily cadence for } 2 \text { seasons, } R<20.5 \mathrm{mag} \\
\text { DOT, daily cadence for } 2 \text { seasons, } R<22 \mathrm{mag}\end{array}$ \\
\hline Lens confirmation & Spectroscopy & $2 \times 600 s$ & 4-m class, $\mathcal{R} \geq 200, R \leq 20 \mathrm{mag}$ \\
\hline $\begin{array}{l}\text { Microlensing } \\
\text { Microlensing }\end{array}$ & $\begin{array}{l}\text { Multi-band imaging } \\
\text { Spectroscopy }\end{array}$ & $\begin{array}{l}4 \times 320 \mathrm{~s} / \mathrm{band} \\
2 \times 600 \mathrm{~s}\end{array}$ & $\begin{array}{l}\geq 2 \text {-m class; optical } \rightarrow \text { NIR; single or multi-epoch } \\
4 \text {-m class, multi-epoch, } \mathcal{R}>300\end{array}$ \\
\hline
\end{tabular}

The latter enables the separation of the different emission components, each subject to a different amount of microlensing. Spectroscopy covering optical and NIR wavelengths at a single-epoch is however more challenging to achieve. Alternatively, a more precise characterization of the AGN structure may be derived by monitoring spectroscopically a lensed AGN over several years. The best constraints may be obtained by monitoring large magnification events (e.g. Eigenbrod et al. 2008b, Mediavilla et al. 2015, Braibant et al. 2017), for which the optical continuum emission is amplified by several tenths of magnitude over a period of several months to several years. The exact duration and amplitude of the event depends on the source size and effective transverse velocity of the lens (see e.g. Mosquera 2011). For an event reaching a magnification of 0.5 mag over a period of about one year, a weekly cadence is considered as optimal. A signal-to-noise ratio larger than 100 is desired for the continuum emission (integrated over a small wavelength range). To study emission line deformations induced by microlensing a signal-to-noise ratio larger then 10 per spectral element is needed.

\section{Summary}

In the upcoming era of large surveys (e.g. Euclid, Large Synoptic Survey Telescope; Laureijs et al. 2011, LSST Science Collaboration 2009) and very large telescopes (e.g. Extremely Large Telescope, Thirty Meter Telescope; Gilmozzi and Spyromilio 2007, Sanders 2013), 2- to 8-m class optical telescopes will play an important role to select the most interesting targets for follow-up studies, but may also enable specific scientific programmes unachievable with larger telescopes that may offer only few possibilities to carry out time-consuming programs. We have identified several important science cases, associated to the observation of strongly-lensed AGNs, for which the DOT and other BINA telescope may play a crucial role. This encompasses (1) the photometric monitoring of lensed systems to measure the expansion rate of the Universe $\left(H_{0}\right),(2)$ the spectroscopic confirmation of lensed candidates and measurement of the lens and source redshifts, and (3) the characterization of the structure of AGNs through microlensing. Table 1 summarizes the main technical requirements for the success of such programs. We stress that since the multiple images of a strongly-lensed AGN are separated by typically less than a few arcseconds, it will be required to systematically observe them under good seeing conditions (typically seeing $<1.2^{\prime \prime}$ ), in order to be able to deblend the lensed components. This required seeing may be accompanied with a good spatial sampling of the image/spectrum (typically better than $0.35^{\prime \prime} /$ pixel). The existence of an adaptive optics system, e.g. the SALTO system (Orban de Xivry et al. 2019), would therefore be a great asset for such projects.

There are no major technical challenges in carrying-out those programs that could already make 
use of existing instruments mounted on the DOT and other BINA telescopes. However, the most ambitious ones require monitoring capabilities over periods that may reach a few years. This is in our opinion, the main challenge to face, but also the most rewarding since the time domain is still poorly exploited in extragalactic astronomy. While the future operation of the Large Synoptic Survey Telescope will play a key role in the time domain for imaging, it may allow one to measure high accuracy time delays only in a small fraction of strongly-lensed AGNs (Liao et al. 2015). On the other hand, spectroscopic monitoring of lensed quasars has been rarely attempted, despite its important scientific impact.

\section{Acknowledgements}

This work is supported in part by ERC project COSMICLENS-787886.

\section{References}

Abbott B. P., Abbott R., Abbott T. D. et al. 2017, Natur, 551, 85

Agnello A., Kelly B. C., Treu T., Marshall P. J. 2015, MNRAS, 448, 1446

Birrer S., Treu T., Rusu C. E. et al. 2019, MNRAS, 484, 4726

Blackburne J. A., Kochanek C. S. 2010, ApJ, 718, 1079

Bonvin V., Tewes M., Courbin F. 2016, A\&A, 585, A88

Bonvin V., Courbin F., Suyu S. H. et al. 2017, MNRAS, 465, 4914

Braibant L., Hutsemékers D., Sluse D., Anguita T. 2016, A\&A, 592, A23

Braibant L., Hutsemékers D., Sluse D., Goosmann R. 2017, A\&A, 607, A32

Chung H., Ramaprakash A. N., Omar A. et al. 2014, SPIE, 9147, id. 91470V

Courbin F., Bonvin V., Buckley-Geer E. et al. 2018, A\&A, 609, A71

Courbin F., Magain P., Kirkove M., Sohy, S. 2000, ApJ, 529, 1136

Delchambre L., Krone-Martins A., Wertz O. et al. 2019, A\&A, 622, A165

Ducourant C., Wertz, O., Krone-Martins A. et al. 2018, A\&A, 618, A56

Edelson R., Gelbord J., Cackett E. et al. 2019, ApJ, 870, 123

Eigenbrod A., Courbin F., Vuissoz C. et al. 2005, A\&A, 436, 25

Eigenbrod A., Courbin F., Dye S. et al. 2006, A\&A, 451, 747

Eigenbrod A., Courbin F., Meylan G. et al. 2008a, A\&A, 490, 933

Eigenbrod A., Courbin F., Sluse D., Meylan G., Agol, E. 2008b, A\&A, 480, 647

Fausnaugh M. M., Starkey D. A., Horne, K. et al. 2018, ApJ, 854, 107

Gao F., Braatz J. A., Reid M. J. et al. 2016, ApJ, 817, 128

Gilmozzi R., Spyromilio, J. 2007, The Messenger, 127, 11

Laureijs R., Amiaux J., Arduini S. et al. 2011, Euclid Definition Study Report, arXiv:1110.3193

Lemon C. A., Auger M. W., McMahon R. G. 2019, MNRAS, 483, 4242

Liao K., Treu T., Marshall P. et al. 2015, ApJ, 800, 11

LSST Science Collaboration: Abell P. A. et al. 2009, LSST Science Book, Version 2.0, arXiv:0912.0201

Magain P., Courbin F., Sohy S. 1998, ApJ, 494, 472

Mediavilla E., Jimenez-Vicente J., Muñoz, J. A., Mediavilla T., Ariza O. 2015, ApJ, 798, 138

Morgan C. W., Kochanek C. S., Morgan N. D., Falco E. E. 2010, ApJ, 712, 1129

Mosquera A. M., Kochanek C. S. 2011, ApJ, 738, 96

Oguri M., Marshall P. J. 2010, MNRAS, 405, 2579

Orban de Xivry G., Absil O., Moreau V. 2019, BSRSL, 88, 55 (this volume)

Refsdal S. 1964, MNRAS, 128, 307

Sanders G. H. 2013, JApA, 34, 81

Sluse D., Hutsemékers D., Anguita T., Braibant L., Riaud P. 2015, A\&A, 582, A109

Sluse D., Hutsemékers D., Courbin F., Meylan G., Wambsganss J. 2012, A\&A, 544, A62

Tie S. S., Kochanek C. S. 2018, MNRAS, 473, 80

Walsh D., Carswell R. F., Weymann R. J. 1979, Natur, 279, 381 